\title{
Corneal Epithelial Remodeling After Femtosecond Laser-Assisted in Situ Keratomileusis Combined with Intraoperative Accelerated Corneal Collagen Crosslinking for Myopia: A Retrospective Study
}

\section{Junjie Piao}

Chinese Academy of Medical Sciences and Peking Union Medical College

\section{Shen Wang}

Beijing Vision Optometry

Ye Tao

Beijing Vision Optometry

Yue Hua Zhou

Chengdu University of Traditional Chinese Medicine

Ying Li (D liyingpumch@126.com)

Chinese Academy of Medical Sciences and Peking Union Medical College

\section{Research Article}

Keywords: LASIK Xtra, myopia, corneal thickness, keratometry, ocular response analyzer

Posted Date: December 2nd, 2021

DOI: https://doi.org/10.21203/rs.3.rs-1128803/v1

License: (c) (1) This work is licensed under a Creative Commons Attribution 4.0 International License. Read Full License 


\section{Abstract \\ Background}

This study analyzed regional corneal thickness remodeling, biomechanical properties, and visual outcomes after femtosecond laser-assisted in situ keratomileusis combined with intraoperative accelerated corneal collagen crosslinking (LASIK Xtra) for myopia.

\section{Methods}

This retrospective study comprised 21 consecutive patients (18 women and 3 men; 42 eyes) who were treated with LASIK Xtra. All treatments were performed with ultraviolet-A (energy, $2.7 \mathrm{~J} / \mathrm{cm}^{2}$; irradiance, 30 $\mathrm{mW} / \mathrm{cm}^{2}$ ), using continuous (90 s) illumination. Postoperative values of corneal biometrics and visual outcomes were compared with preoperative values. Corneal thickness changes were evaluated using anterior segment optical coherence tomography. All patients were followed up for 12 months postoperatively. Preoperative and postoperative data were compared statistically using the paired t-test for normally distributed parameters and the Wilcoxon rank-sum test and Friedman analysis of variance with Bonferroni correction for non-normally distributed data.

\section{Results}

Uncorrected distance visual acuity (UDVA) significantly improved at 6 months after surgery $(P<0.001)$. The central and inner regional corneal epithelial thickness significantly increased after LASIK Xtra $(P<$ 0.05 for all), while peripheral corneal epithelial thickness remained stable at 12 months after surgery. There was also a statistically significant decreased in the stromal thickness at most locations $(P<0.05$ for all), except in the outer superior and outer superior-temporal regions.

\section{Conclusion}

LASIK Xtra provided predictable postoperative UDVA, corneal curvature, and corneal biomechanical stability. Since the retrospective study results depended on the cohort members' past information, it is inferred and confirmed that regular corneal thickness remodeling occurred after treatment.

\section{Background}

Myopia has emerged as a major health issue in Asia, because of the increased use of electronic products and modern living habits. The concerns of patients regarding esthetics, occupational requirements for first responders, athletes, military, media, and other professions, along with the long-term discomfort and infection risk associated with contact lens use [1], have increased the demand for surgical vision 
correction procedures for conditions such as myopia, hyperopia, and irregular astigmatism. However, iatrogenic keratectasia has emerged as a serious complication of laser vision correction surgery [2]. With advancements in diagnosis and femtosecond laser platforms, the incidence of iatrogenic keratectasia has decreased from $0.66-0.021 \%[3,4]$. Risk factors include the amount of tissue altered and therefore, high myopia, unevenly distributed corneal thickness, abnormal corneal topography, hyperopic treatments, weakened corneal biomechanical properties, and thin predicted residual corneal thickness $[2,5]$.

Corneal collagen crosslinking $(\mathrm{CXL})$ is a safe and effective therapeutic technique for halting the progression of keratoconus and iatrogenic keratectasia. Therefore, femtosecond laser-assisted in situ keratomileusis (LASIK) procedures combined with intraoperative accelerated corneal collagen crosslinking (LASIK Xtra) were developed [6,7]. LASIK Xtra has similar safety and efficacy compared with conventional LASIK procedures $[4,8]$.

LASIK procedures using Wavelight FS200 (Alcon Laboratories, Fort Worth, TX, USA) femtosecond laser combined with VISX Star S4 (Abbott Medical Optics, Santa Ana, CA, USA) excimer laser platforms provide predictable, efficacious, and safe postoperative outcomes $[9,10]$. The VISX Star S4 excimer laser system's ablation software has a tendency toward central islands (Cl) formation [11], and the effect of $\mathrm{Cl}$ formation on visual rehabilitation, safety, and stability after LASIK Xtra (energy, $2.7 \mathrm{~J} / \mathrm{cm}^{2}$; irradiance, 30 $\mathrm{mW} / \mathrm{cm}^{2}$ ) has not been fully elucidated. In 2014, an anti-Cl formation software was used with the VISX Star S4 excimer laser, which significantly decreased the rate of $\mathrm{Cl}$ formation and improved postoperative best-corrected distance visual acuity $[11,12]$. Differences in epithelial profiles can mask measures of corneal topography, such as corneal stress concentration directions between the optical zone and the untreated ablation zone [12,13].

LASIK Xtra has significantly reduced the epithelial increase [14] and corneal haze [15] in comparison with LASIK. Moreover, regional differences in corneal epithelial healing occurred after corneal refractive surgery with VISX Star S4 excimer laser. We hypothesize that the corneal epithelial profiles would be regular and remain stable after LASIK Xtra following VISX Star S4 excimer laser combined with accelerated superficial CXL. This retrospective study aimed to clinically evaluate corneal epithelial and stromal remodeling after LASIK Xtra.

\section{Methods}

This retrospective study comprised 21 consecutive patients (42 eyes) diagnosed with moderate to high myopia at the Department of Ophthalmology, Peking Union Medical College Hospital, Beijing, China, between February 2017 and October 2018. The study protocol followed the guidelines of the Declaration of Helsinki and the Institutional Review Board for Human Studies and was approved by the Peking Union Medical College Hospital Institutional Ethics Committee (S-K1768-1). Written informed consent was obtained from all the patients before the study was initiated. 
Inclusion criteria for the study were as follows: borderline corneal topography (defined as 1 diopter [D] or greater inferior steepening in some areas but an inferior-superior value of less than 1.4 D or unevenly distributed corneal thickness) or high myopia with thin cornea [16], potential to improve postoperative refractive stability regarding myopic regression, and therefore lower risk of iatrogenic keratectasia. All patient were aged $>18$ years and had no other ocular pathologic signs (such as ocular surface infection or allergy). Exclusion criteria included history of ocular surgery or of conditions such as diabetes, autoimmune or endocrine pathologies, dry eye symptoms, insufficient follow-up, re-treatment of accelerated $\mathrm{CXL}$, and pregnancy or lactation.

\section{Surgical procedures}

All procedures were performed by an experienced surgeon (Y.L.). After topical anesthesia with $0.5 \%$ proparacaine (Alcaine, Alcon-Couvreur; Puurs-Sint-Amands, Belgium, USP), a corneal flap was created using VisuMax (Carl Zeiss Meditec AG, Jena, Germany) femtosecond laser platforms. With a superior hinge, an intended flap diameter of $8.5 \mathrm{~mm}$, a flap thickness of 90-95 $\mu \mathrm{m}$, and corneal ablation was performed using the VISX S4IR excimer laser (Abbott Medical Optics, Santa Ana, CA, USA). Then, the corneal bed was saturated with a solution of $0.22 \%$ dextran-free riboflavin ophthalmic solution in normal saline (Vibex Xtra ${ }^{\mathrm{TM}}$, Avedro, Waltham, MA, USA), which was at the center of the cornea and allowed to soak for $90 \mathrm{~s}$. After complete soaking of the riboflavin, the solution was rinsed from the eye with a $0.9 \%$ balanced saline solution. Next, UVA energy was applied at $2.7 \mathrm{~J} / \mathrm{cm}^{2}$ with an irradiance of $30 \mathrm{~mW} / \mathrm{cm}^{2}$ to the eye that underwent continuous (90 s) light illumination for LASIK Xtra (KXL system; Avedro, Inc., Waltham, MA, USA) (Table A). Finally, the eye was rinsed again with a $0.9 \%$ balanced saline solution, and a bandage contact lens (PureVision; Bausch \& Lomb, Rochester, NY, USA) was applied to the cornea until complete re-epithelialization was achieved.

After surgery, all patients were prescribed topical $0.5 \%$ levofloxacin 4 times daily for 2 weeks, $0.5 \%$ loteprednol etabonate (Lotemax; Bausch \& Lomb, Tampa, FL, USA), and preservative-free artificial tears (Hycosan; Hylo-Comod, Ursapharm Arzneimittel, Germany) 4 times daily for 1 month, and $0.2 \%$ carbomer eye gel (Liposic; Bausch \& Lomb, Brunsbütteler Damm, Berlin, Germany) once daily at night for 1 month.

\section{Outcome measures}

Examinations were performed preoperatively and postoperatively and at 1 and 3 days; 1 week; and 1, 3, 6, and 12 months postoperatively. Slit-lamp examination, best spectacle-corrected visual acuity with and without a pinhole, uncorrected distance visual acuity (UDVA), corrected distance visual acuity (CDVA) using a logarithm of the minimum angle of resolution (logMAR) chart with tumbling $E$, corneal topography (TMS-4N; TOMEY, Erlangen, Germany), and dual Scheimpflug imaging (Gallilei; Ziemer Ophthalmology, Port, Switzerland), and ultrasonic pachymetry (TOMEY Ltd, Aichi, Japan) of the central cornea were performed. Goldmann applanation tonometry and an ocular response analyzer (Reichert Technologies; Depew, NY, USA) were used to measure intraocular pressure (IOP) and corneal biomechanical properties. The corneal demarcation line and the corneal epithelial and stromal 
thicknesses were measured and evaluated using anterior segment optical coherence tomography (ASOCT) (Optovue RTVue XR, Optovue; Fremont, CA, USA). Corneal epithelial and stromal thickness profiles were obtained at the thinnest part of the central cornea using 16 peripheral measurements on the corneal vertex, and measurements and statistical analyses of the central 6-mm zone (inner areas, $3 \mathrm{~mm}$ zone of corneal vertex; outer areas, $6 \mathrm{~mm}$ zone of corneal vertex) were performed [17]. For ultrasound pachymetry, the average measurements of the corneal thickness values were chosen (each single measurement represented the mean of 5 consecutive measurements). Postoperative evaluation included UDVA, corneal biomechanical parameters, corneal topography, and corneal thickness profiles (epithelia and stroma) using AS-OCT.

\section{Statistical analysis}

Data regarding all the evaluated parameters were recorded in a Microsoft Excel spreadsheet (Microsoft; Redmond, WA, USA), and statistical analysis was performed using SPSS for Mac (version 25.0; IBM Corp., Armonk, New York, USA). Data normality was tested using the Shapiro-Wilk test, and a paired t-test was performed to analyze changes between preoperative and postoperative data. If the data were not normally distributed, the Wilcoxon rank-sum test was performed to analyze changes between preoperative and postoperative data. Friedman analysis of variance with the Bonferroni correction was applied for repeated parameter measurements at the 12 months follow-up, in case data did not show a normal distribution. Statistical significant was set at $P<0.05$.

\section{Results}

Forty-two consecutive eyes underwent bilateral LASIK Xtra. This study included 18 women (86\%) and 3 men (14\%). The mean age was $25.76 \pm 5.35$ years, and the mean preoperative UDVA and CDVA were 1.38 $\pm 0.23 \log M A R$ and $-0.01 \pm 0.03 \log M A R$, respectively. The preoperative manifest refractive spherical equivalent was $-7.28 \pm 2.35 \mathrm{D}$. Demographic details and refractive outcomes are presented in Table 1 .

\section{Epithelial and stromal remodeling}

The preoperative and postoperative corneal epithelial and stromal thickness changes after 12 months are summarized in Table 2. There was a statistically significant thickened in the central and inner regional corneal epithelial thickness due to remodeling during the 12-month follow-up ( $P<0.05$ for all). The outer regional epithelium was thickened at 6 months postoperatively and repopulated at 12 months postoperatively ( $P>0.05$ for all). There was a statistically significant thinning in the stromal thickness profiles in most locations $(P<0.05$ for all). The comparison of the differences between preoperative and postoperative values are shown in Figure 1A and Figure 1B.

\section{Refractive outcomes and corneal biomechanical properties}

The changes in visual acuity, corneal curvature, IOP (such as corneal compensated IOP [IOPcc] and goldmann-correlated IOP [IOPg]), and corneal biomechanical properties (such as corneal hysteresis [CH] 
and corneal resistance factor [CRF]) after 12 months are summarized in Table 3. All the parameters showed statistically significant differences between the preoperative and postoperative follow-up values $(P<0.05$ for all).

\section{Discussion}

Corneal surface ablation surgery has the potential to affect corneal biomechanical properties and increase the incidence of iatrogenic keratectasia when considerable corneal volume is removed or a corneal flap is created. On the contrary, the main goal of the accelerated, superficial CXL fluence is to regulate the elaborate system for epithelial-stromal communication through increase in cytokines and growth factors to maintain corneal strength without inducing a refractive change. According to the results of the present study, significant epithelial remodeling occurred after LASIK Xtra, with a variation in epithelial profiles, fluence corneal power, and biomechanical properties compared to those seen preoperatively.

Corneal hyperopic ablation is the preferred tissue removal procedure for the innately thicker paracentral cornea, and it may partially account for the rarity of iatrogenic keratectasia and higher estimated postoperative $\mathrm{CH}$ and $\mathrm{CRF}$ values compared to myopic correction, which ablates the central cornea to steepen the paracentral cornea [18]. Therefore, the corneal biomechanical properties were weakened after refractive surgery; they may have influenced the IOP values during the follow-up period. In the current study, IOPcc and IOPg were found to have statistically significant reductions after treatment. Flap creation during LASIK surgery causes disruption of the peripheral collagen fibers and damages the components of the extracellular matrix (ECM); therefore, ECM repair may affect $\mathrm{CH}$ and CRF values [19]. In this study, $\mathrm{CH}$ and $\mathrm{CRF}$ values were found to be stable after treatment.

Corneal ectasia arises from changes in the amount of collagen fibrils and interfibrillar substances in the stromal layer. However, the corneal stromal fibroblast dysfunction disrupts epithelial cell-cell junctions and leads to epithelial thinning and ulceration [20]. Therefore, timely detection and monitoring of corneal epithelial profiles are becoming more important in the preclinical diagnosis of iatrogenic keratectasia [21]. During the follow-up at 12 months after the surgery, significant inner regional epithelial remodeling occurred after LASIK Xtra, and a doughnut-like profile with central thickened epithelium was observed after treatment. Kanellopoulos et al. [14] reported that the postoperative epithelial thickness increased significantly in the mid-peripheral region by $3.79 \mu \mathrm{m}$ and $3.95 \mu \mathrm{m}$ for the "-8.00 D to $-9.00 \mathrm{D}$ " and "-7.00 D to $-8.00 \mathrm{D}$ " after LASIK Xtra, compared to $9.75 \mu \mathrm{m}$ and $7.14 \mu \mathrm{m}$ after LASIK alone, for the same degree of myopia. These results confirmed the hypothesis that differences in epithelial and stromal thickness indicate corneal ECM repair after LASIK Xtra. Shih et al. [22] reported corneal stress concentration in an oblique downward direction after corneal refractive surgery. According to our results, the steepest and flattest meridian keratometry values were $5.44 \pm 1.89 \mathrm{D}$ and $4.97 \pm 1.79 \mathrm{D}$ at 6 months postoperatively, while the corresponding values at 12 months postoperatively were $5.32 \pm 1.82 \mathrm{D}$ and $4.92 \pm 1.68 \mathrm{D}$. A similar refractive shift has been reported in another study [23]. 
The refractive outcomes were stable 12 months after treatment, with good results in terms of UDVA, which is consistent with the decrease in the initial corneal curvature commonly reported after LASIK Xtra $[8,23]$. Kohnen et al. [8] demonstrated that postoperative UDVA significantly improved at 1 week postoperatively, but did not show significant differences between the values obtained with LASIK Xtra and LASIK, from $0.08 \pm 0.14 \log M A R$ and $0.04 \pm 0.13 \log M A R$ to $-0.02 \pm 0.15 \log M A R$ and $0.01 \pm 0.15$ logMAR after 12 months. Deep corneal haze observed after conventional CXL differs from the subepithelial haze after corneal refractive surgery, with the latter having less effect on the corneal fibroblasts. Aslanides et al. [15] demonstrated that corneal surface ablation combined with accelerated $\mathrm{CXL}$ has the same effect on postoperative corneal haze in long-term follow-up as that with $0.02 \%$ mitomycin C (MMC) following corneal refractive surgery. Eyes that underwent LASIK Xtra had an equal or better visual acuity than eyes that underwent stand-alone LASIK [8,23], and $90 \%$ of eyes achieved $20 / 40$ or better UDVA after LASIK Xtra in the current study.

The limitations of our study include the possible effect of a single-center retrospective clinical study, a 12 months follow-up period, small sample size of patients undergoing bilateral LASIK Xtra, and the 6-mm corneal diameter limit for epithelial remodeling. AS-OCT provides a high-quality imaging of 6-mm corneal thickness profile, which is a clinical quantitative and qualitative investigation of corneal epithelial and stromal remodeling after surgery. Kanellopoulos et al. [14] found a statistically significant reduction in epithelial increase after LASIK Xtra $\left(30 \mathrm{~mW} / \mathrm{cm}^{2}\right.$ for a total of $\left.80 \mathrm{~s}\right)$ compared to that after stand-alone LASIK procedures. However, flap creation and CXL are affected by central corneal thicknesses greater than $6 \mathrm{~mm}$. Future cohort studies should involve a larger sample size and allow for imaging of corneal diameters up to $10 \mathrm{~mm}$, which would cover the entire area affected by LASIK flap creation and CXL. Such studies would provide a greater insight into the entire area of epithelial remodeling.

\section{Conclusions}

LASIK Xtra not only provided an equally CXL-like effect on corneal anterior stromal fibroblast activity in strengthening corneal biomechanical properties, but also contributes to an MMC-like effect on decreasing corneal haze formation [15]. In addition, the superior postoperative UDVA and postoperative corneal biomechanical stability could potentially lead to more definite changes in corneal curvature. However, detection and monitoring of epithelial remodeling profile maps would be a more sensitive measurement tool than the assessment of surface topography or stromal surface changes induced by LASIK Xtra.

\section{Abbreviations}

LASIK, femtosecond laser-assisted in situ keratomileusis; LASIK Xtra, LASIK procedures combined with intraoperative accelerated corneal collagen crosslinking; logMAR, logarithm of the minimum angle resolution; $\mathrm{CXL}$, corneal collagen crosslinking; $\mathrm{Cl}$, central islands; UDVA, uncorrected distance visual acuity; CDVA, corrected distance visual acuity; IOP, intraocular pressure; IOPcc, compensated intraocular pressure; IOPg, goldmann-correlated intraocular pressure; AS-OCT, anterior segment optical coherence 
tomography; $\mathrm{CRF}$, corneal resistance factor; $\mathrm{CH}$, corneal hysteresis; $\mathrm{ECM}$, extracellular matrix; $\mathrm{MMC}$, mitomycin $\mathrm{C}$.

\section{Declarations}

\section{Ethics approval and consent to participate}

The study protocol followed the guidelines of the Declaration of Helsinki and the Institutional Review Board for Human Studies and was approved by the Peking Union Medical College Hospital Institutional Ethics Committee (S-K1768-1). Written and informed consent was obtained from all the participants before the study was initiated.

\section{Consent for publication}

Not applicable.

\section{Availability of data and materials}

Available from the first author on reasonable request.

\section{Competing interests}

The authors declare that they have no competing interests.

\section{Funding}

No Funding.

\section{Authors' contributions}

Study concept and design: ZYH and YL; Data collection; JP, WS and TY; Analysis and interpretation of data: JP, WS, and TY; Writing the manuscript: JP; Critical revision of the manuscript: JP; Statistical expertise: JP, WS and TY; Administrative, technical, or material support: WS, TY, ZYH, and YL; Supervision: WS, TY, ZYH, and YL. All the authors have read and approved the final manuscript.

\section{Acknowledgements}

None.

\section{Authors' information}

${ }^{1}$ Department of Ophthalmology, Peking Union Medical College Hospital, Chinese Academy of Medical Sciences \& Peking Union Medical College, Beijing 100730, China. ${ }^{2}$ Department of Medical Science Research Center, Translational Medicine Center, Peking Union Medical College Hospital, Chinese Academy of Medical Sciences and Peking Union Medical College, Beijing 100730, China. ${ }^{3}$ Beijing Vision Optometry, 
Beijing 100191, China. ${ }^{4}$ College of Ophthalmology, Chengdu University of Traditional Chinese Medicine, Chengdu 610075, Sichuan, China.

\section{References}

1. Konne NM, Collier SA, Spangler J, Cope JR. Healthy contact lens behaviors communicated by eye care providers and recalled by patients - United States, 2018. MMWR Morb Mortal Wkly Rep. 2019;68:693-7.

2. Ambósio R Jr. Post-LASIK ectasia: twenty years of a conundrum. Semin Ophthalmol. 2019;34:66-8.

3. Jin SX, Dackowski E, Chuck RS. Risk factors for postlaser refractive surgery corneal ectasia. Curr Opin Ophthalmol. 2020;31:288-92.

4. Ortege-Usobiaga J, Llovet-Osuna F, Djodeyre MR, Llovet-Rausell A, Beltran-Sanz J, Baviera-Sabater J. Post-lasik corneal ectasia in patients with significant differences in keratometry readings between both eyes. Arch Soc Esp Oftalmol. 2014;89:99-103.

5. Chan C, Saad A, Randleman JB, Harissi-Dagher M, Chua D, Qazi M, et al. Analysis of cases and accuracy of 3 risk scoring systems in predicting ectasia after laser in situ keratomileusis. $J$ Cataract Refract Surg. 2018;44:979-92.

6. Ziaei M, Gokul A, vellara H, Patel D, McGhee CNJ. Prospective two year study of changes in corneal density following transepithelial pulsed, epithelium-off continuous and epithelium-off pulsed, corneal crosslinking for keratoconus. Cont Lens Anterior Eye. 2020;43:458-64.

7. Ağca A, Tülü B, Dilek Y, Kepez Yıldız B, Sucu ME, Genç S, et al. Accelerated corneal crosslinking in children with keratoconus: 5-year results and comparison of 2 protocols. $J$ Cataract Refract Surg. 2020;46:517-23.

8. Kohnen T, Lwowski C, Hemkeppler E, de'Lorenzo N, Petermann K, Forster R, et al. Comparison of femto-LASIK with combined accelerated cross-linking to femto-LASIK in high myopic eyes: a prospective randomized trial. Am J Ophthalmol. 2020;211:42-55.

9. Meidani A, Tzavara C. Comparison of efficacy, safety, and predictability of laser in situ keratomileusis using two laser suites. Clin Ophthalmol. 2016;10:1639-46.

10. Reed DS, Apsey D, Steigleman W, Townley J, Caldwell M. Retrospective analysis of the post-operative changes in higher-order aberrations: a comparison of the Wavelight EX500 to the VISX S4 laser in refractive surgery. Mil Med. 2017;182:e2061-5.

11. Kamiya K, Takahashi M, Igarashi A, Shoji N. Central islands and visual outcomes of phototherapeutic keratectomy using the photorefractive keratectomy mode. Cornea. 2019;38:89-92.

12. Junjie Piao, Li YJ, Whang WJ, Choi M, Kang MJ, Lee JH, et al. Comparative evaluation of visual outcomes and corneal asphericity after laser-assisted in situ keratomileusis with the six-dimension Amaris excimer laser system. PLoS One. 2017;12:e0171851.

13. Piao J, Whang WJ, Joo CK. Comparison of visual outcomes after femtosecond laser-assisted LASIK versus flap-off epipolis LASIK for myopia. BMC Ophthalmol. 2020;20:310. 
14. Kanellopoulos AJ, Asimellis G. Epithelial remodeling after femtosecond laser-assisted high myopic LASIK comparison of stand-alone with LASIK combined with prophylactic high-fluence cross-linking. Cornea. 2014;33:463-9.

15. Aslanides IM, Hafezi F, Chen S, Mukherjee H, Selimis V, Maragkos I, et al. 5-year efficacy of all surface laser ablation with cross-linking (ASLA-XTRA) for the treatment of myopia. Eye Vis (Lond). 2020;7:31.

16. Randleman JB, Woodward M, Lynn MJ, Stulting RD. Risk assessment for ectasia after corneal refractive surgery. Ophthalmology. 2008;115:37-50.

17. Atia R, Jouve L, Sandali O, et al. Early epithelial remodeling after standard and iontophoresis-assisted corneal cross-linking as evaluated by spectral-domain optical coherence tomography. J Refract Surg. 2018;34:551-8.

18. de Medeiros FW, Sinha-Roy A, Alves MR, Wilson SE, Dupps WJ Jr. Differences in the early biomechanical effects of hyperopic and myopic laser in situ keratomileusis. J Cataract Refract Surg. 2010;36:947-53.

19. Wu D, Wang Y, Zhang L, Wei S, Tang X. Corneal biomechanical effects: small-incision lenticule extraction versus femtosecond laser-assisted laser in situ keratomileusis. J Cataract Refract Surg. 2014;40:954-62.

20. Gabison EE, Huet E, Baudouin C, Menashi S. Direct epithelial-stromal interaction in corneal wound healing: role of EMMPRIN/CD147 in MMPs induction and beyond. Prog Retin Eye Res. 2009;28:1933.

21. Rapuano CJ. Prevention of iatrogenic keratectasia. Klin Monbl Augenheilkd. 2016;233:695-700.

22. Shih PJ, Wang IJ, Cai WF, Yen JY. Biomechanical simulation of stress concentration and intraocular pressure in corneas subjected to myopic refractive surgical procedures. Sci Rep. 2017;7:13906.

23. Kanellopoulos AJ, Asimellis G, Karabatsas C. Comparison of prophylactic higher fluence corneal cross-linking to control, in myopic LASIK, one year results. Clin Ophthalmol. 2014;8:2373-81.

\section{Tables}

Table A. LASIK Xtra methods 


\begin{tabular}{|c|c|}
\hline Parameter & LASIK Xtra \\
\hline Treatment target & Myopia \\
\hline Fluence (total) $\left(\mathrm{J} / \mathrm{cm}^{2}\right)$ & 2.7 \\
\hline Soak time and interval (s) & 90 \\
\hline Intensity (mW) & 30 \\
\hline Treatment time (s) & 90 \\
\hline Epithelium status & Off \\
\hline Chromophore & $\begin{array}{l}\text { Riboflavin solution in normal saline (Vibex } \mathrm{Xtra}^{\mathrm{TM}} \text {, Avedro, Waltham, MA, } \\
\text { USA) }\end{array}$ \\
\hline Chromophore carrier & Dextran-free \\
\hline Chromophore osmolarity & Iso-Osmolar \\
\hline $\begin{array}{l}\text { Chromophore } \\
\text { concentration }\end{array}$ & $0.22 \%$ \\
\hline Light source & UV-A light (KXL system; Avedro, Inc., Waltham, MA, USA) \\
\hline $\begin{array}{l}\text { Irradiation mode } \\
\text { (interval) }\end{array}$ & Continuous \\
\hline Protocol modifications & None \\
\hline
\end{tabular}

Table 1. Preoperative Patient Demographic Data 


\begin{tabular}{ll} 
Parameter & LASIK Xtra \\
\hline Eyes/Patients (n) & $42 / 21$ \\
\hline Age (y) & $25.76 \pm 5.35$ \\
\hline Sex (M/F) & $3 / 18$ \\
\hline MRSE (D) & $-7.28 \pm 2.35$ \\
\hline Ks (D) & $44.68 \pm 1.51$ \\
\hline AvgK (D) & $44.01 \pm 1.44$ \\
\hline Cyl (D) & $1.34 \pm 0.85$ \\
\hline UDVA (logMAR) & $1.38 \pm 0.23$ \\
\hline CDVA (logMAR) & $-0.01 \pm 0.03$ \\
\hline $\begin{array}{l}\text { LASIK Xtra = femtosecond laser-assisted in situ keratomileusis combined with intraoperative } \\
\text { accelerated corneal collagen crosslinking; } y=\text { years; MRSE = manifest refraction spherical equivalent; }\end{array}$ \\
$\begin{array}{l}\text { Ks = steepest keratometry; AvgK = front average keratometry; Cyl = keratometric astigmatism; D = } \\
\text { diopters; UDVA = uncorrected distance visual acuity; CDVA = corrected distance visual acuity; logMAR } \\
=\text { logarithm of the minimum angle of resolution }\end{array}$
\end{tabular}

Table 2. Mean Corneal Thickness Changes After LASIK Xtra 
Mean \pm SD

\begin{tabular}{|c|c|c|c|c|}
\hline Parameter & Pre-op & 6-month Post-op & 12-month Post-op & $P$ value \\
\hline \multicolumn{5}{|l|}{ Stromal thickness $(\mu \mathrm{m})$} \\
\hline Central & $496.22 \pm 22.94$ & $424.39 \pm 27.03^{*}$ & $429.11 \pm 26.04^{\star}$ & $<0.001$ \\
\hline Inner Inferior & $507.72 \pm 23.32$ & $461.17 \pm 21.49 *$ & $464.83 \pm 23.92^{\star}$ & $<0.001$ \\
\hline Inner Inferior-nasal & $512.44 \pm 23.98$ & $464.28 \pm 26.22^{\star}$ & $448.11 \pm 94.66^{\star}$ & $<0.001$ \\
\hline Inner Nasal & $518.22 \pm 24.22$ & $469.83 \pm 29.03^{\star}$ & $475.50 \pm 29.55^{\star}$ & $<0.001$ \\
\hline Inner Superior-nasal & $528.56 \pm 24.44$ & $483.00 \pm 28.54^{\star}$ & $485.50 \pm 29.70^{\star}$ & $<0.001$ \\
\hline Inner Superior & $530.78 \pm 24.29$ & $489.50 \pm 28.47^{\star}$ & $492.56 \pm 25.93^{\star}$ & $<0.001$ \\
\hline Inner Superior-temporal & $520.22 \pm 23.26$ & $477.11 \pm 27.47^{\star}$ & $478.56 \pm 25.10^{\star}$ & $<0.001$ \\
\hline Inner Temporal & $506.94 \pm 23.62$ & $461.67 \pm 25.48^{\star}$ & $462.83 \pm 23.70^{*}$ & $<0.001$ \\
\hline Inner Inferior-temporal & $504.11 \pm 23.09$ & $458.83 \pm 21.50 *$ & $460.17 \pm 22.51^{\star}$ & $<0.001$ \\
\hline Outer Inferior & $536.57 \pm 28.09$ & $512.17 \pm 18.80^{*}$ & $511.00 \pm 25.84^{\star}$ & 0.016 \\
\hline Outer Inferior-nasal & $533.22 \pm 24.41$ & $516.83 \pm 25.77^{\star}$ & $516.44 \pm 30.19 *$ & 0.023 \\
\hline Outer Nasal & $538.44 \pm 24.84$ & $525.67 \pm 28.24^{\star}$ & $525.11 \pm 36.98$ & 0.090 \\
\hline Outer Superior-nasal & $545.72 \pm 24.70$ & $543.83 \pm 24.45$ & $535.33 \pm 44.19$ & 0.114 \\
\hline Outer Superior & $560.67 \pm 25.75$ & $554.72 \pm 25.97$ & $549.22 \pm 33.33$ & 0.220 \\
\hline Outer Superior-temporal & $568.39 \pm 23.29$ & $531.83 \pm 30.09$ & $528.22 \pm 33.49$ & 0.100 \\
\hline Outer Temporal & $528.28 \pm 23.27$ & $504.56 \pm 30.25^{\star}$ & $502.94 \pm 31.22$ & 0.050 \\
\hline Outer Inferior-temporal & $525.44 \pm 23.12$ & $504.39 \pm 20.27^{\star}$ & $507.39 \pm 30.98^{*}$ & 0.023 \\
\hline \multicolumn{5}{|l|}{ Epithelial thickness $(\mu \mathrm{m})$} \\
\hline Central & $53.11 \pm 3.20$ & $67.06 \pm 16.90^{*}$ & $61.72 \pm 5.51^{\star}$ & $<0.001$ \\
\hline Inner Inferior & $53.83 \pm 3.24$ & $65.00 \pm 17.98^{*}$ & $61.17 \pm 4.68^{\star}$ & $<0.001$ \\
\hline Inner Inferior-nasal & $53.83 \pm 3.09$ & $67.67 \pm 15.51^{*}$ & $60.28 \pm 4.16^{\star}$ & $<0.001$ \\
\hline Inner Nasal & $53.11 \pm 2.74$ & $62.94 \pm 11.84^{\star}$ & $58.11 \pm 4.74^{\star}$ & $<0.001$ \\
\hline Inner Superior-nasal & $53.06 \pm 3.06$ & $62.00 \pm 14.59 *$ & $57.50 \pm 4.67 *$ & $<0.001$ \\
\hline Inner Superior & $52.61 \pm 3.13$ & $63.11 \pm 19.35^{\star}$ & $55.11 \pm 12.09 *$ & 0.001 \\
\hline Inner Superior-temporal & $52.28 \pm 2.91$ & $66.33 \pm 16.97 *$ & $59.11 \pm 4.14^{\star}$ & $<0.001$ \\
\hline Inner Temporal & $52.44 \pm 3.57$ & $63.17 \pm 10.65^{\star}$ & $60.44 \pm 4.02^{\star}$ & $<0.001$ \\
\hline
\end{tabular}




\begin{tabular}{|c|c|c|c|c|}
\hline Inner Inferior-temporal & $52.89 \pm 3.76$ & $64.22 \pm 11.82^{\star}$ & $60.67 \pm 4.73^{\star}$ & $<0.001$ \\
\hline Outer Inferior & $54.11 \pm 3.29$ & $57.39 \pm 17.17$ & $53.78 \pm 6.45$ & 0.780 \\
\hline Outer Inferior-nasal & $53.78 \pm 3.04$ & $59.22 \pm 15.49$ & $52.50 \pm 5.29$ & 0.397 \\
\hline Outer Nasal & $53.44 \pm 2.75$ & $54.67 \pm 11.54$ & $51.72 \pm 6.23$ & 0.478 \\
\hline Outer Superior-nasal & $52.83 \pm 3.67$ & $56.00 \pm 12.72$ & $51.50 \pm 5.15$ & 0.462 \\
\hline Outer Superior & $51.72 \pm 3.34$ & $58.00 \pm 17.99$ & $51.56 \pm 5.68$ & 0.629 \\
\hline Outer Superior-temporal & $51.56 \pm 2.87$ & $60.44 \pm 17.14$ & $53.89 \pm 6.46$ & 0.415 \\
\hline Outer Temporal & $51.89 \pm 3.69$ & $57.28 \pm 12.30$ & $53.67 \pm 6.91$ & 0.467 \\
\hline Outer Inferior-temporal & $52.78 \pm 3.66$ & $57.39 \pm 12.88$ & $53.67 \pm 7.63$ & 0.871 \\
\hline \multicolumn{5}{|c|}{$\begin{array}{l}\text { LASIK Xtra = femtosecond laser-assisted in situ keratomileusis combined with intraoperative } \\
\text { accelerated corneal collagen crosslinking; } \mathrm{SD}=\text { standard deviations; } \mu \mathrm{m}=\text { micrometer; } \text { Pre-op = } \\
\text { preoperative; Post-op = postoperative }\end{array}$} \\
\hline
\end{tabular}

Table 3. Changes in UDVA, corneal curvature, IOP and corneal biomechanical parameters After LASIK Xtra 


\begin{tabular}{|c|c|c|c|}
\hline \multicolumn{4}{|c|}{ Mean \pm SD } \\
\hline Parameter & Pre-op & 6-month Post-op & 12-month Post-op \\
\hline UDVA (logMAR) & $1.38 \pm 0.23$ & $0.04 \pm 0.08^{\star}$ & $0.05 \pm 0.09 *$ \\
\hline Ks (D) & $44.68 \pm 1.51$ & $39.24 \pm 2.08 *$ & $39.36 \pm 2.04^{*}$ \\
\hline$K f(D)$ & $43.35 \pm 1.50$ & $38.38 \pm 1.93^{\star}$ & $38.43 \pm 1.82^{\star}$ \\
\hline AvgK (D) & $44.01 \pm 1.44$ & $38.81 \pm 1.99 *$ & $38.85 \pm 1.96^{*}$ \\
\hline Cyl (D) & $1.34 \pm 0.85$ & $0.86 \pm 0.46 *$ & $0.92 \pm 0.48 *$ \\
\hline IOPcc (mmHg) & $15.46 \pm 3.33$ & $13.71 \pm 2.20$ * & $13.80 \pm 1.85^{\star}$ \\
\hline $\mathrm{IOPg}(\mathrm{mmHg})$ & $13.21 \pm 3.40$ & $9.34 \pm 2.16$ & $9.37 \pm 2.33^{*}$ \\
\hline $\mathrm{CRF}(\mathrm{mmHg})$ & $8.63 \pm 1.37$ & $6.16 \pm 1.26^{\star}$ & $6.12 \pm 1.38 *$ \\
\hline $\mathrm{CH}(\mathrm{mmHg})$ & $9.09 \pm 1.19$ & $7.58 \pm 1.21^{\star}$ & $7.51 \pm 1.12^{\star}$ \\
\hline \multicolumn{4}{|c|}{$\begin{array}{l}\text { LASIK Xtra = femtosecond laser-assisted in situ keratomileusis combined with intraoperative } \\
\text { accelerated corneal collagen crosslinking; Pre-op = preoperative; Post-op = postoperative; UDVA = } \\
\text { uncorrected distance visual acuity; Ks = steepest keratometry; Kf = flattest keratometry; AvgK = fron } \\
\text { average keratometry; Cyl = keratometric astigmatism; IOPcC = corneal compensated intraocular } \\
\text { pressure; IOPg = goldmann-correlated intraocular pressure; CRF = corneal resistance factor; } \mathrm{CH}= \\
\text { corneal hysteresis; } \mathrm{mmHg}=\mathrm{mill} \text { limeters of mercury; logMAR = logarithm of the minimum angle of } \\
\text { resolution; } \mathrm{D}=\text { diopters; } \mathrm{mmHg}=\text { millimeters of mercury }\end{array}$} \\
\hline$\star P$ value was sta & ignificant dif & between postope & baseline. \\
\hline
\end{tabular}

\section{Figures}




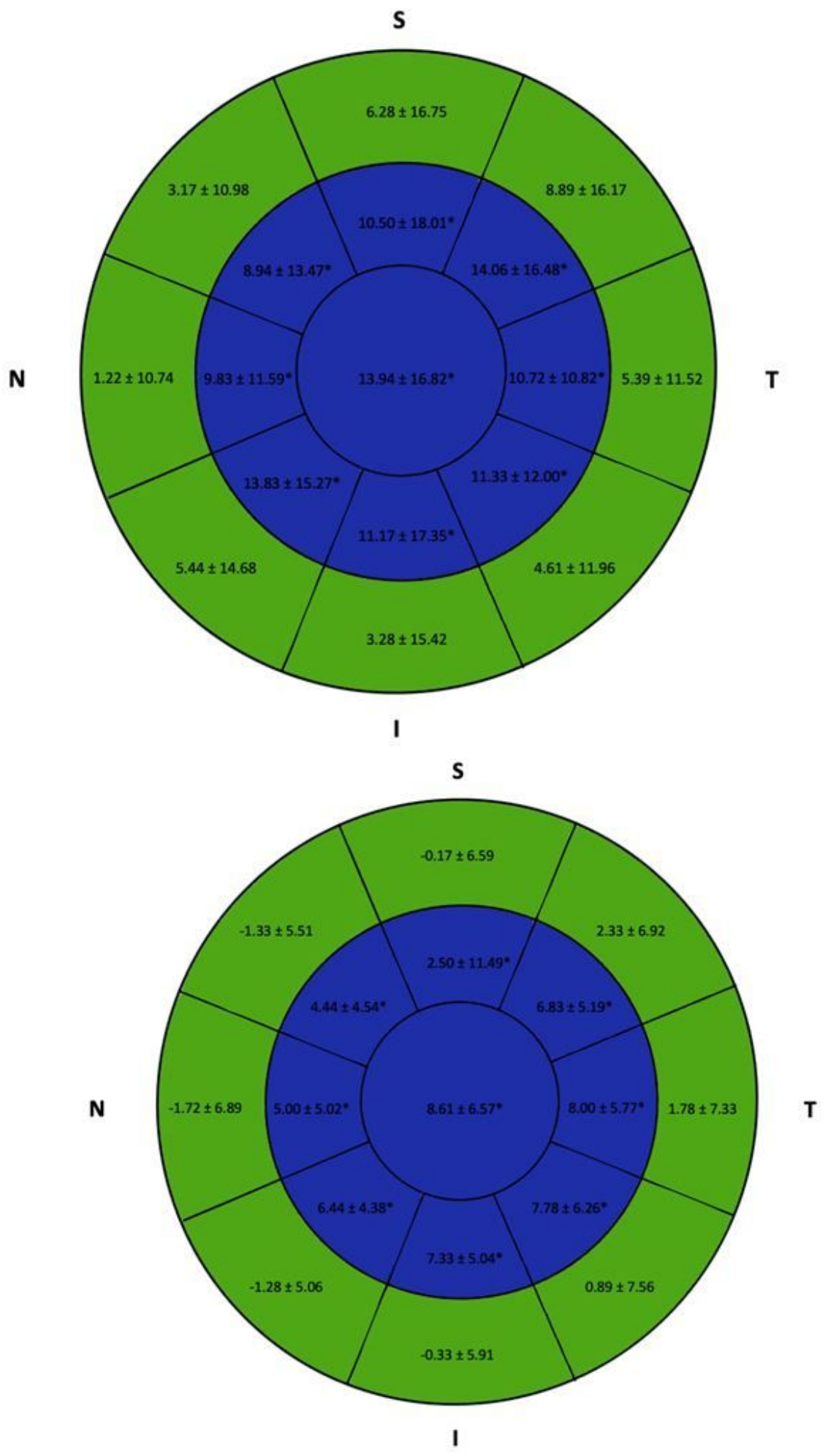

\section{Figure 1}

Mean changes in epithelial thickness distribution after femtosecond laser-assisted in situ keratomileusis combined with intraoperative accelerated corneal collagen crosslinking during the 12 months follow-up (A. 6 months postoperatively; B. 12 months postoperatively). Values in blue indicate a significant change after surgery $(\mathrm{P}<0.05)$. S: superior; l: inferior; $\mathrm{T}$ : temporal; $\mathrm{N}$ : nasal. 